J. Clin. Chem. Clin. Biochem.

Vol. 21, 1983, pp. 673-678

\title{
Nachweis der Qualitätssteigerung von Harnsteinanalysen anhand von Ergebnissen aus 7 Ringversuchen zur externen Qualitätskontrolle \\ Qualitätsverbesserung von Harnsteinanalysen durch Schaffung einheitlicher methodischer Voraussetzungen in den Harnsteinanalyse-Laboratorien, 2. Teil.
}

\author{
Von G. Rebentisch \\ Zentrallaboratorium des Bezirkskrankenhauses Cottbus und \\ W. Berg \\ Harnsteinlabor der Urologischen Klinik der Friedrich-Schiller-Universität Jena
}

(Eingegangen am 28. Februar/9. Juni 1983)

Zusammenfassung: 7 Qualitätskontroll-Ringversuche bestätigen die Brauchbarkeit der standardisierten Harnsteinanalyse mittels Röntgendiffraktion. Mit einem vorgeschlagenen Bewertungsschema zur Beurteilung der Ringversuchsergebnisse sind vergleichbare qualitative Betrachtungen zwischen den Analysezentren möglich. Das Qualitätsmaß verbesserte sich nach Anwendung der standardisierten Analysevorschrift maßgebend. Die Qualitätsvorteile der röntgendiffraktometrischen Harnsteinanalyse gegenüber polarisationsmikroskopischer, infrarotspektroskopischer, differentialthermoanalytischer und naßchemischer Analysentechnik werden anhand der Ringversuchsergebnisse eines internationalen Teilnehmerkreises deutlich.

Evidence of improved urolith analysis from seven quality control surveys

Improvement in the quality of urolith analysis by the establishment of uniform methodology in urolith analysis laboratories, Part 2

Summary: Seven quality control surveys confirmed the usefulness of standardized urolith analysis by X-ray diffraction. A method is suggested for tabulating the results of the surveys, which permits qualitative comparison of participating analysis centres. Quality improved considerably after the introduction of the standardized instructions for analysis. The results of the survey with international participants clearly demonstrated the superiority of X-ray diffractometric urolith analysis over polarization microscopical, infrared spectroscopic, differential thermoanalytical and wet chemical analytical techniques.

\section{Einfiihirung}

In den Jahren 1977 bis 1982 wurden in den Harnsteinanalysezentren der DDR zentral durch Einwaage bereitete Harnsteinproben mit 1-4 Komponenten in regelmäßig durchgeführten Ringversuchen analysiert. Die Teilnahme erfolgte auf freiwilliger Basis. Jeder Ringversuch bestand aus 5 Analysenproben. Ab Ringversuch III (1980) arbeiteten alle Analysezentren nach der inzwischen standardisierten röntgendiffraktometrischen Analysenmethode $(1,2)$.
Die vereinheitlichte Methodik erlaubt über ein vorgeschlagenes Auswerteprogramm eine problemlose Vergleichbarkeit der Analysenergebnisse und gleichsam die Aufdeckung und Eliminierung von Analysenfehlern. Somit ist eine Möglichkeit zur kontinuierlichen externen Qualitätskontrolle geschaffen worden, deren Ergebnisse sich in positiver Weise auf therapeutische Maßnahmen bei der Behandlung der Urolithiasis auswirken sollten. 


\section{Material und Methode}

Material und Methodik sind im vorangehenden Beitrag (3) beschrieben.

\section{Ergebnisse und Diskussion}

\section{Qualitätskontroll-Ringversuche}

Bereits 1977 und 1978 waren 2 orientierende Qualitätskontroll-Ringversuche durchgeführt worden, an denen sich 9 Analysezentren beteiligten (Tab. 1) (4).

An dem 1980 durchgeführten III. Ringversuch nahmen 7 von 10 Harnsteinanalysezentren der DDR teil. Aus zentral bereiteten Gemischen (Bezugssubstanz: $\mathrm{MgO}$ ) wurden zunächst in jedem Zentrum die Spektren aufgenommen, aus denen dann die Bezúgsfaktoren für die Auswertepeaks der Bezugssubstanzen unter den jeweiligen standardisierten Gerätebedingungen errechnet werden konnten. Úber die Bezugsfaktoren waren 5 zentral bereitete Harnsteinproben unbekannter Zusammensetzung zu analysieren. Trotz stark differierender substanz- und gerätespezifischer Faktoren zur Auswertung nach dem Konstantenverfahren ergab sich eine sehr gute Übereinstimmung der Ergebnisse aller teilnehmenden Harnsteinanalysenzentren (Tab. 1).

Generell wurde erkannt, daß es sich um 1 Dreikomponenten- und 4 Zweikomponentengemische handelte. Ebenso wurden alle vorhandenen Harnsteinsubstanzen richtig beurteilt, keine zusätzlichen Substanzen angegeben und in allen Fällen die entsprechende Hauptkomponente zugeordnet. Die mittlere Zusammensetzung kommt der Sollzusammensetzung sehr nahe. Größere Schwankungen offenbaren lediglich die Analysenergebnisse des Carbonatapatit/Struvit-Gemisches. Alle anderen Gemische zeigen akzeptable Streuungen bei minimaler Abweichung von der Soll-Konzentration, die pro Komponente zwischen 0,01 und 0,09 Mol-Anteilen liegt.

Beim Ringversuch IV im Frühjahr 1981 kamen 3 Dreistoff- und 2 Zweistoffgemische mit analytisch anspruchsvoller Zusammensetzung zur Auswertung. Probleme traten durch den Koinzidenzreichtum im System Harnsäure/Harnsäuredihydrat/Monoammoniumurat und im System Carbonatapatit/Struvit auf.

Am Ringversuch V im Herbst 1981 beteiligten sich erstmals 12 Analysenzentren. Die Auswertung läßt bei allen Untersuchern eine sehr gute Übereinstimmung der Soll- und mittleren gefundenen Zusammensetzung erkennen.

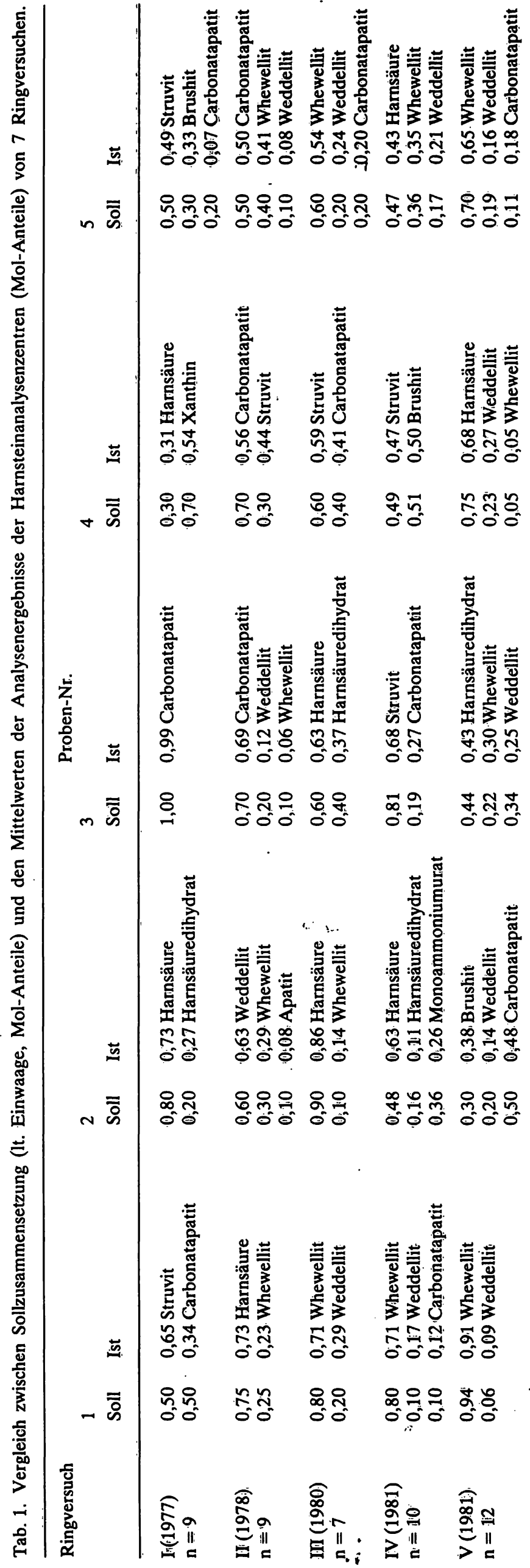

J. Clin. Chem. Clin. Bịochẹm. / Vol. 21, 1983 / No. 11 


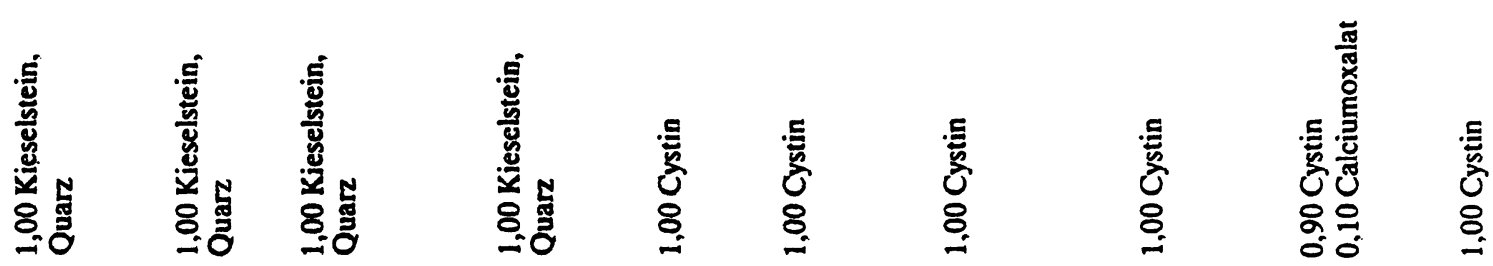

8

8
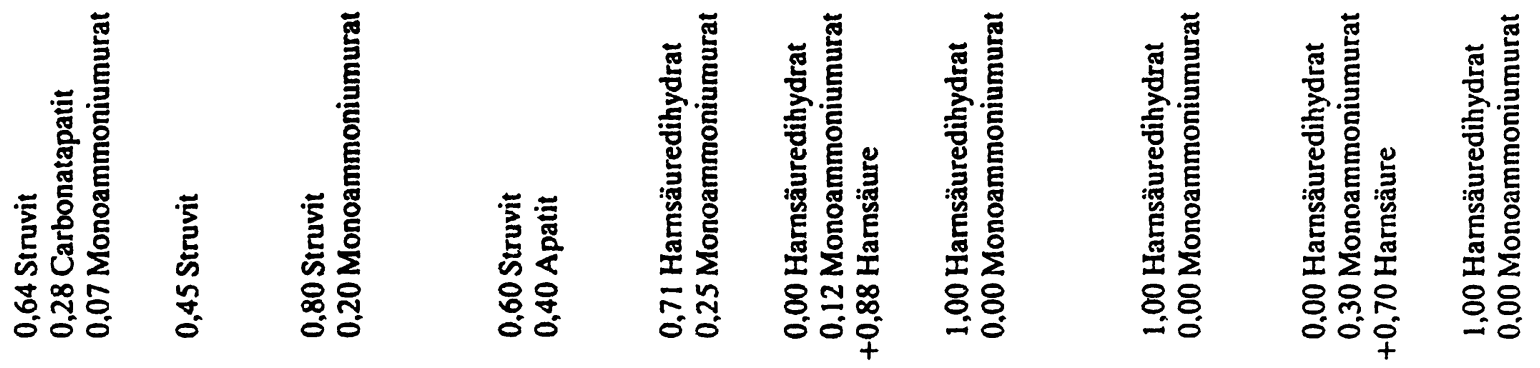

ํํㅇ정동

ำ
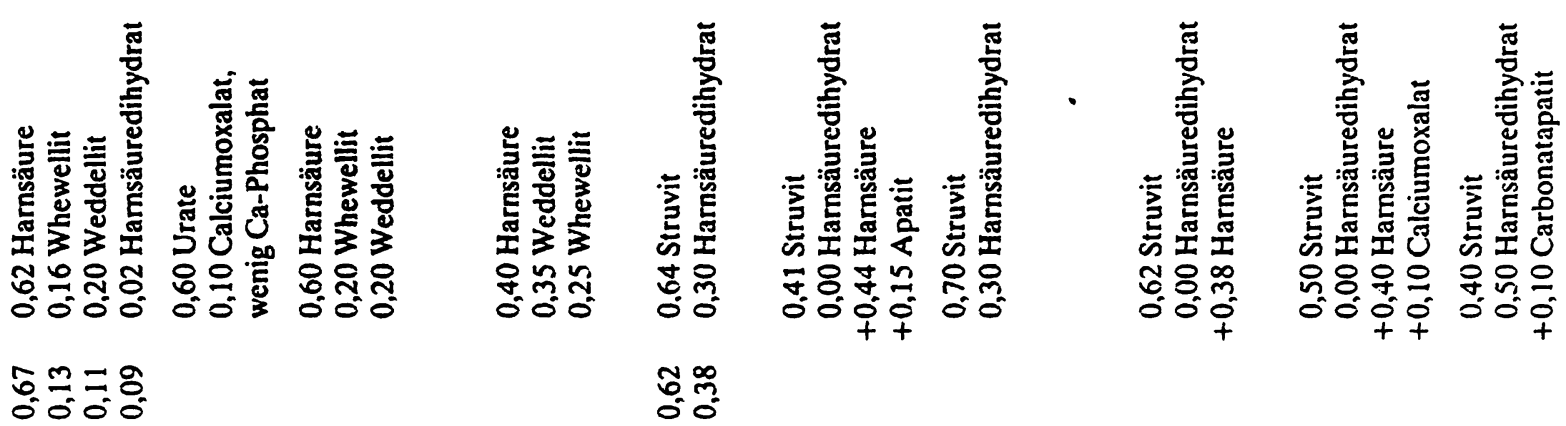
웅웅
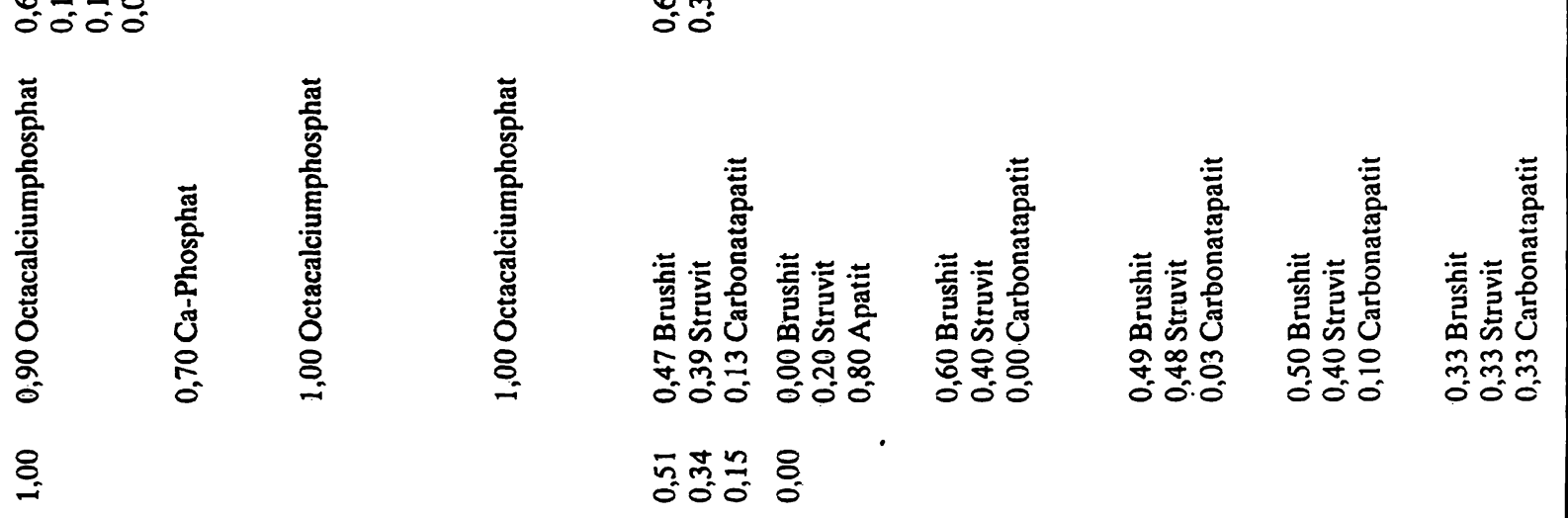

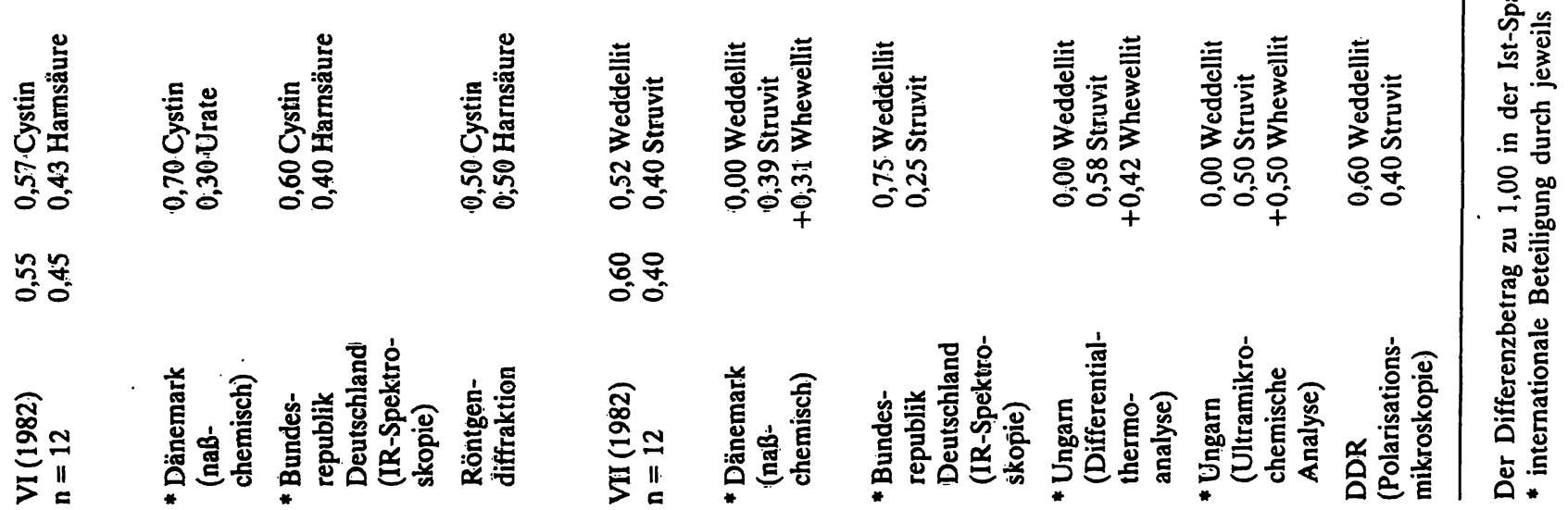


Der im Jahre 1982 durchgeführte Ringversuch VI bestand aus jeweils einem Vierstoff-, Dreistoff- und Zweistoffgemisch sowie zwei Einphasensystemen, von denen eines eine harnsteinatypische Substanz $\left(\mathrm{SiO}_{2}\right)$ enthielt. Letztere wurde ausnahmslos als solche erkannt. Stellt man die Sollzusammensetzungen den Analysemittelwerten für die Ringversuche I-VI gegenüber, ergeben sich nach Tabelle 1 folgende Aussagen:

1. Die durchschnittliche Abweichung von der Sollzusammensetzung beträgt pro Komponente 0,05 Mol-Anteile

2. Maximale Abweichungen bis zu 0,15 Mol-Anteilen treten in den Problem-Gemischen Carbonatapatit/Struvit und Harnsäure/Harnsäuredihydrat/Monoammoniumurat auf.

3. Schwierig scheint der Nachweis kleiner Mol-Anteile von Harnsäuredihydrat neben viel Harnsäure und wenig Weddellit sowie der Nachweis von Monoammoniumurat neben Carbonatapatit und Struvit zu sein.

Es wurde der Versuch unternommen, für die 7 durchgeführten Ringversuche ein Bewertungsschema auf der Grundlage eines Punktsystems aufzustellen. Úber ein Qualitätsmaß (QM) sollten die einzelnen Harnsteinanalysenzentren noch besser vergleichbar sein. Der Punkteschlüssel variiert dabei mit der Anzahl der Komponenten im Gemisch.

Bewertet wurden:

1. die Erkennung der richtigen Anzahl und der tatsächlich vorhandenen Harnsteinphasen,

2. die Klassifikation nach Haupt- und Nebenbestandteilen,

3. die Abweichung von der Sollzusammensetzung pro Komponente.

Verfolgt wurde der Entwicklungsstand der Analysequalität vor und nach Standarrdisierung der röntgendiffraktometrischen Harnsteinanalyse, die eine einheitliche Auswertung über das Konstantenverfahren (3) zugrundelegt. Als Bewertungsschema kam der in der Tabelle 2 niedergelegte Punktmodus zur Anwendung.

Tab. 2. Qualitäts-Bewertungsschema.

Bei monomineralischer Zusammensetzung:

1. Erkennung der Anzahl der Komponenten

a) 1 Komponente richtig erkannt 0 Komponente richtig erkannt

b) 0 Komponente zusätzlich erkannt

1 Komponente zusätzlich erkannt

2 Komponenten zusätzlich erkannt
Tab. 2. Fortsetzung.

\begin{tabular}{|c|c|c|}
\hline 2. & Punkte & Qualitätsmaß \\
\hline & 3 & 1 \\
\hline & 2 & 2 \\
\hline & 1 & 3 \\
\hline & 0 & 4 \\
\hline
\end{tabular}

Für binäre Systeme:

,

1. Erkennung der Anzahl der Komponenten

a) 2 Komponenten richtig erkannt 1 Komponente richtig erkannt 0 Komponente richtig erkannt

2 Punkte 1 Punkt

0 Punkte

b) 0 Komponente zusätzlich erkannt

1 Komponente zusätzlich erkanñnt

2 Komponenten zusätzlich erkannt

3 Komponenten zusätzlich erkannt

3 Punkte

2 Punkte

1 Punkt

0 Punkte

2. Haupt-/Nebenbestandteil (bei A $>$ B)

$\begin{array}{llll}A>B & 2 \text { Punkte } & \text { B }>\text { A } & \text { 1 Puñkt } \\ A=B & \text { 1 Punkt } & \text { B } & \text { 0 Punkte } \\ A \geqslant C & \text { 1 Punkt } & \text { C } & \text { 0 Punkte }\end{array}$

$A>B>C \quad 1$ Punkt

A 1 Punkt

3. Durchschnittliche Abweichung von der Sollzusammensetzung.

$\leqslant \pm 0,10$ Mol-Anteile

$> \pm 0,10 \leqslant \pm 0,20$ Mol-Anteile

$> \pm 0,20$ Mol-Anteile

2 Puñkte

1 Punkt

4.

$\begin{array}{ll}\text { Punkte } & \text { Qualitätsmaß } \\ 9 & 1 \\ 8 & 2 \\ 6=7 & 3 \\ 4-5 & 4 \\ 0-3 & 5\end{array}$

Für Dreikomponentensysteme:

1. Anzahl der Komponenten

a) 3 Komponenten richtig erkannt 3 Punkte 2 Komponenten richtig erkannt 2 Punkte 1 Komponente richtig erkannt " $": \cdot \quad 1$ Punkt

0 Komponenten richtig erkannt 0 Punkte

b) 0.Komponenten zusätzlich erkannt 3 Punkte

1 Komponente zusätzlich erkannnt 2 Punkikte

2 Komponenten zusätżlich erkannt 1 Punkt

3 Komponenten zusätzlich erkannt $\quad$ O Punkte

2. Haupt-/Nebenbestandteil (bei $\mathrm{A}>\mathrm{B}>\mathrm{C}$ )

\begin{tabular}{|c|c|c|c|}
\hline $\begin{array}{l}A>B \geqslant C \\
A=B=\dot{C} \\
A>B=C\end{array}$ & $\begin{array}{l}3 \text { Punkte } \\
3 \text { Punkte } \\
3 \text { Punkte }\end{array}$ & $\begin{array}{l}A>C \geqslant B \\
A=C>B \\
B>A>C \\
A \geqslant B \\
A \geqslant C \\
B \geqslant A\end{array}$ & $\begin{array}{l}2 \text { Punkte } \\
2 \text { Punkte } \\
2 \text { Punkte } \\
2 \text { Punkte } \\
2 \text { Punkte } \\
2 \text { Punkte }\end{array}$ \\
\hline $\begin{array}{l}C>A>B \\
B>C \geqslant A \\
B \geqslant C\end{array}$ & $\begin{array}{l}1 \text { Punkt } \\
1 \text { Punkt } \\
1 \text { Punkt }\end{array}$ & $\begin{array}{l}C>B>A \\
C \geqslant A \\
C \geqslant B\end{array}$ & $\begin{array}{l}\text { 0 Punkte } \\
\text { 0 Punkikte } \\
\text { O Punkinte }\end{array}$ \\
\hline
\end{tabular}

3. Durchschnittliche Abweichung von der Sollzusammensetzung

$\leqslant \pm 0,10 \mathrm{Mol}-$ Anteile

$> \pm 0,10 \leqslant \pm 0,20$ Mol-Anteile 1 Punkt

$> \pm 0,20$ Mol-Anteile

0 Punkte

\begin{tabular}{ccc} 
4. & Punkte & Qualitätsmaß \\
11 & 1 & \\
$9-10$ & 2 \\
$7-8$ & 3 & \\
$5-6$ & 4 & \\
$0-4$ & 5 & $\therefore$ \\
\hline
\end{tabular}


Als Ergebnis läßt sich aus Tabelle 3 ableiten:

1. Die Teilnehmerzahl an den Ringversuchen wuchs von 9 auf 12 an.

2. Das relativ schlechte Qualitätsmaß der lediglich von einem Analysezentrum praktizierten IRAnalysetechnik dürfte durch die hier fehlende Standardisierung sowie schwierig zu führende exakte Ermittlung der quantitativen Zusammensetzung bedingt sein (Zentrum 9). Auch ist offenbar noch mangelnde Erfahrung in der praktischen Spektrenauswertung bei einigen Einrichtungen Grund für ein relativ unbefriedigendes Qualitäts$\mathrm{maß}$ (Zentrum 11).

3. Generell verbessert sich das Qualitätsmaß mit Anwendung der standardisierten röntgendiffraktometrischen Harnsteinanalyse von 2,25 auf 1,75. Dabei werden auch in den erst später einbezogenen Einrichtungen (Zentren 6 und 8) durchschnittlich gute Ergèbnisse erhalten.

Die deutliche Steigerung der Analysenqualität wurde erreicht durch:

1. eine einheitliche Analysetechnik,

2. Bereitstellung einheitlicher Probengemische aus Harnsteinsubstanz und $\mathrm{MgO}$ als Inertsubstanz zur Faktorenanalyse,
3. Anwendung des Konstantenverfahrens zur quantitativen Phasenanalyse und

4. regelmäßige Durchführung von Ringversuchen mit kontinuierlicher Auswertung und Erläuterung analytischer Möglichkeiten sowie Erfahrungsaustausch im Rahmen der „AG Urolithiasis" der Gesellschaft für Urologie der DDR (in Verantwortung der Jenaer Leiteinrichtung).

Die im Ringversuch VII mit erweiterter internationaler Beteiligung durch naßchemische, polarisationsmikroskopische, IR-spektroskopische und Differentialthermo-Analysetechnik erhaltenen Ergebnisse verweisen auf folgende methodisch bedingte Probleme:

\section{Naßchemische Methode}

Die Angabe „Urate" anstelle Harnsäure und Harnsäuredihydrat führt $\mathrm{zu}$ falschen therapeutischen Konsequenzen. Das Nichterkennen von Harnsäuredihydrat .und Identifizierung desselben als Harnsäure liegt in den begrenzten Aussagemöglichkeiten der Methode begründet. Ähnliches ist zum Verwechseln von Whewellit und Weddellit zu sagen. Calciumphosphat anstelle Carbonatapatit und Nichterkennen von Monoammoniumurat neben Struvit und Carbonatapatit sind Analysefehler.

Tab. 3. Trends in der Entwicklung der Qualität der Harnsteinanalysen in den Harnsteinanalysenzentren der DDR anhand von bisher durchgeführten Ringversuchen.

\begin{tabular}{|c|c|c|c|c|c|c|c|c|c|c|c|c|c|c|c|c|}
\hline \multirow{3}{*}{$\begin{array}{l}\text { Harn- } \\
\text { stein- } \\
\text { ana- } \\
\text { lysen- } \\
\text { zentrum }\end{array}$} & $\begin{array}{l}\text { Rin } \\
\text { I } \\
197\end{array}$ & ersuch & $\begin{array}{l}\text { II } \\
197\end{array}$ & & & $\begin{array}{l}\text { Rir } \\
\text { III } \\
198\end{array}$ & ersuch & \multicolumn{2}{|c|}{$\begin{array}{l}\text { IV } \\
1981\end{array}$} & \multicolumn{2}{|c|}{$\begin{array}{l}V \\
1981\end{array}$} & \multicolumn{2}{|c|}{$\begin{array}{l}\text { VI } \\
1982\end{array}$} & \multicolumn{2}{|c|}{$\begin{array}{l}\text { VII } \\
1982\end{array}$} & \multirow{3}{*}{$\begin{array}{l}\overline{\mathrm{QM}} \\
1980-82\end{array}$} \\
\hline & \multicolumn{2}{|c|}{$\begin{array}{l}\text { Soll-P. } \\
41\end{array}$} & \multicolumn{2}{|c|}{$\begin{array}{l}\text { Soll-P. } \\
51\end{array}$} & & \multicolumn{2}{|c|}{$\begin{array}{l}\text { Soll-P. } \\
47\end{array}$} & \multicolumn{2}{|c|}{$\begin{array}{l}\text { Soll-P. } \\
51\end{array}$} & \multicolumn{2}{|c|}{$\begin{array}{l}\text { Soll-P. } \\
53\end{array}$} & \multicolumn{2}{|c|}{$\begin{array}{l}\text { Soll-P. } \\
39\end{array}$} & \multicolumn{2}{|c|}{$\begin{array}{l}\text { Soll-P. } \\
41\end{array}$} & \\
\hline & $\mathbf{P}$ & QM & $\mathbf{P}$ & Q̀M & & $\mathbf{P}$ & $\mathbf{Q M}$ & $\mathbf{P}$ & $\mathbf{Q M}$ & $\mathbf{P}$ & $\mathrm{QM}$ & $\mathbf{P}$ & $\mathrm{QM}$ & $\mathbf{P}$ & $\mathrm{QM}$ & \\
\hline 1 & 41 & 1 & 51 & 1 & 1,0 & 46 & 1 & 50 & 1 & 53 & 1 & 37 & 1 & 40 & 1 & 1,0 \\
\hline 2 & 31 & 2 & 42 & 2 & 2,0 & 47 & 1 & 51 & 1 & 51 & 1 & 33 & 2 & 41 & 1 & 1,2 \\
\hline 3 & 37 & 2 & 38 & 3 & 2,5 & 44 & 1 & 45 & 2 & 48 & 2 & 38 & 1 & 39 & 1 & 1,4 \\
\hline 4 & 39 & 1 & 43 & 2 & 1,5 & 46 & 1 & 44 & 2 & 52 & 1 & 33 & 2 & 37 & 2 & 1,6 \\
\hline 5 & 36 & 2 & 39 & 2 & 2,0 & 46 & 1 & 39 & 2 & 43 & 2 & 34 & 2 & 36 & 2 & 1,8 \\
\hline 6 & - & - & $=$ & - & $=$ & 47 & 1 & 46 & 2 & 38 & 3 & 30 & 3 & 33 & 2 & 2,2 \\
\hline 7 & 30 & 3 & 46 & 2 & 2,5 & 47 & 1 & 41 & 2 & 47 & 2 & 34 & 2 & 33 & 2 & 1,8 \\
\hline 8 & - & - & - & - & - & - & - & 39 & 2 & 43 & 2 & 32 & 2 & 33 & 2 & 2,0 \\
\hline 9 (IR) & 30 & 3 & 37 & 3 & 3,0 & - & - & - & - & 44 & 2 & 28 & 3 & 37 & 2 & 2,3 \\
\hline 10 & 11 & 4 & 41 & 2 & 3,0 & - & - & 47 & 1 & 53 & 1 & 33 & 2 & 37 & 2 & 1,5 \\
\hline 11 & - & - & - & - & - & - & - & - & - & 41 & 2 & 26 & 3 & 28 & 3 & 2,7 \\
\hline 12 & 25 & 3 & 41 & 2 & 2,5 & - & - & 45 & 2 & 51 & 1 & 34 & 2 & 39 & 1 & 1,5 \\
\hline$\overline{\mathbf{x}}$ & & & & & 2,25 & & & & & & & & & & & 1,75 \\
\hline
\end{tabular}

QM: Qualitätsmaß $\quad \overline{\mathrm{QM}}$ : mittleres QM $\quad$ P: Punkte 
2. Die Polarisationsmikroskopie ist zwar qualitativ recht aussagekräftig, doch können quantitative Angaben zur Zusammensetzung von Harnstein-Substanzgemischen nur Ergebnisse einer Schätzung sein. Problembehaftet scheint die Erkennung von Monoammoniumurat neben Harnsäuredihydrat zu sein.

3. Bei IR-spektroskopischen Analysen ergeben sich in Abhängigkeit von der technischen Ausrüstung der Geräte Probleme in der Quantifizierung der Ergebnisse. Die aus der DDR vorliegenden IR-Harnsteinergebnisse sind Abschätzungen der Mol-Anteile von einzelnen Harnsteinsubstanzen in Gemischen. Mit an IR-Geräte gekoppelten Rechnern läßt sich unter Nutzung spezieller Programme wie Search (5, 6) bei automatischer Spektrenauswertung der Auswerteaufwand minimieren und die Ergebnisfindung objektivieren. Bei komplizierter aufgebauten Mehrkomponentengemischen existieren aber auch hierbei Schwierigkeiten. Der Anteil nicht erkannter Komponenten im Vergleich zur Röntgendiffraktion ist unter Zugrundelegung von Ringversuchsauswertungen in der Bundesrepublik Deutschland doppelt so hoch $(7,8)$. Am Beispiel unserer Ringversuche VI und VII stellen sich Probleme im Nichterkennen von Carbonatapatit neben Struvit und Monoammoniumurat, von Harnsäuredihydrat neben Harnsäure, Whewellit und Weddellit und der Fehlanalyse von Monoammoniumurat neben Harnsäuredihydrat dar.

Bei der Differentialthermoanalyse von HarnsteinSubstanzgemischen treten wider Erwarten Schwierigkeiten in der Differenzierung der unterschiedli- chen Hydratstufen von Calciumoxalat und Harnsäure auf.

5. Bei der ultramikrochemischen Analyse sind die gleichen Probleme wie bei der Differentialthermoanalyse $\mathrm{zu}$ beobachten.

An den Qualitätsringversuchen zur 'Harnsteinanalyse in der Bundesrepublik Deutschland $(7,8)$ beteiligten sich 114 Laboratorien im Jahr 19.80, im Jahr 1981 waren es 63, in Dänemark (9) waren es 54 Einrichtungen. Dabei kamen kommerzielle Testbestekke; wie „Merckognost" und „Oxford Stone Analysis Set" sowie selbstgefertigte Reagenzien für die vorrangig naßchemisch geführten Analysen neben zahlenmäßig gering eingesetzter IR- und Röntgendiffraktionstechnik zur Anwendung. In Auswertung dieser Ringversuche ist die Fehlerquote bei der Vielzahl der Analytiker, aber der doch recht einfachen Analysenzusammensetzung noch relativ hoch und nimmt mit steigender Anzahl der in Gemischproben vorliegenden Komponenten sehr stark zu $(7,8)$. Es wird jedoch übereinstimmend eingeschätżt, $\mathrm{daß}$ der röntgendiffraktometrischen Analysentechnik hinsichtlich ihrer Richtigkeit ein sehr hoher Stellenwert beigemessen werden kann $(7,8,10,11)$. Das bezeugt auch die bisher erfolgreiche Teilnahme der Analyṣezentren 1 und 2 an den Ringversuchen der Bundesrepublik Deutschland und Dänemark.

Die diskutierten Ergebnisșe unterstreichen die Leistungsfähigkeit, Präzision und Richtigkeit der vorgestellten zentralisierten und standardisierten röntgendiffraktometrischen Harnsteinangalyse.

\section{Literatur}

1. Schneider, H.-J., Berg, W., Rebentisch, G., Göthe, W. \& Yersin, A. (1981) Zbl. Pharm. 120, 19-26.

2. Arzneimittelbuch der DDR, Diagnostische Labormethoden (D. L.) Akademie-Verlag, Berlin 1983 (im Druck).

3. Rebentisch, G. \& Berg, W. (1983) J. Clin. Chem. Clin. Biochem. 21, 665-671.

4. Rebentisch, G., Braun, H. \& Schroda, K.-D. (1978) V. Jenaer Harnsteinsymposium, Wissenschaftliche Beiträge der Friedrich-Schiller-Universität Jena, S. 92-95.

5. Hesse, A. \& Moldt, K. (1982) J. Clin. Chem. Clin. Biochem. 20, 861-873.

6. Hesse, A. \& Bach, D. (1982) Harnsteine - Pathobiochemie und klinisch-chemische Diagnostik, Thieme Stuttgart.

7. Röhle, G., Voigt, U., Hesse, A. \& Breuer, H. (1982) J. Clin. Chem. Clin. Biochem. 20, 851-859.

8. Hesse, A., Röhle, G. \& Voigt, U. (1982) Fortschr. Urol. Nephrol. 17, 306-310.

9. Uldall, A. (1981) Scand, J. Clin. Lab. Invest. 41, 339-345.

10. Dosch, W. \& Altrock, K. (1978) Akt. Urol. 5, 105-122.

11. Takasaki, E. (1971) Calc. Tissue Res. 7, 232 240 .

Dr. rer. nat. G. Rebentisch
Zentrallaboratoriưm
des Bezirkskrankenhauses Cottbus
Thiemstraße 111
DDR-7500 Cottbus
Dr. rer. nat. W. Berg
Utologische Klinik
der Friedrich-Schiller-Universität Jena
Lessingstraße 1
DDR=6900 Jena

